\title{
Local visual homing by matched-filter descent in image distances
}

\author{
Ralf Möller • Andrew Vardy
}

Received: 15 February 2005 / Accepted: 14 June 2006 / Published online: 26 September 2006

(C) Springer-Verlag 2006

\begin{abstract}
In natural images, the distance measure between two images taken at different locations rises smoothly with increasing distance between the locations. This fact can be exploited for local visual homing where the task is to reach a goal location that is characterized by a snapshot image: descending in the image distance will lead the agent to the goal location. To compute an estimate of the spatial gradient in the distance measure, its value must be sampled at three noncollinear points. An animal or robot would have to insert exploratory movements into its home trajectory to collect these samples. Here we suggest a method based on the matched-filter concept that allows one to estimate the gradient without exploratory movements. Two matched filters - optical flow fields resulting from translatory movements in the horizontal plane - are used to predict two images in perpendicular directions from the current location. We investigate the relation to differential flow methods applied to the local homing problem and show that the matched-filter approach produces reliable homing behavior on image databases. Two alternative methods that only require a single matched filter are suggested. The matched-filter concept is also applied to derive a home-vector equation for a Fourier-based parameter method.
\end{abstract}

R. Möller $(\varangle)$

Computer Engineering, Faculty of Technology,

Bielefeld University, 33594 Bielefeld, Germany

URL: www.ti.uni-bielefeld.de

A. Vardy

Computer Science/Engineering and Applied Science,

Memorial University of Newfoundland,

St. John's, Canada A1B 3X5

\section{Introduction}

Local visual homing is the ability of an agent to return to a target location by relating the currently perceived visual information to stored visual information taken at the target location. Visual homing methods received attention from neuroethology as models for the navigation abilities of social insects and from robotics as computationally cheap building blocks for topological approaches to map building and navigation (Franz et al. 1998a). Since these methods have been extensively reviewed in recent publications (Vardy and Möller 2005; Zeil et al. 2003; Franz and Mallot 2000), we restrict ourselves to the brief classification shown in Fig. 1. We leave aside methods based on depth information since they require multiple cameras, special sensors (Stürzl and Mallot 2002), or dedicated movement strategies. Methods where only image intensity is available often derive themselves from the snapshot hypothesis of insect visual homing (Wehner and Räber 1979; Cartwright and Collett 1983), according to which insects store a relatively unprocessed "snapshot" image of the surroundings of the target location (be it a nest entrance or a food source) and later return to the target location by moving in such a way that the current view gradually becomes more similar to the snapshot.

Homing methods based on intensity information can be coarsely classified into correspondence methods and holistic methods. Correspondence methods establish correspondences between local regions in snapshot and current views. Each correspondence is expressed by a shift vector describing the movement of the region from one image to another. The shift vector can then be transformed into a direction of movement that would reduce the shift. Averaging a number of these movement 


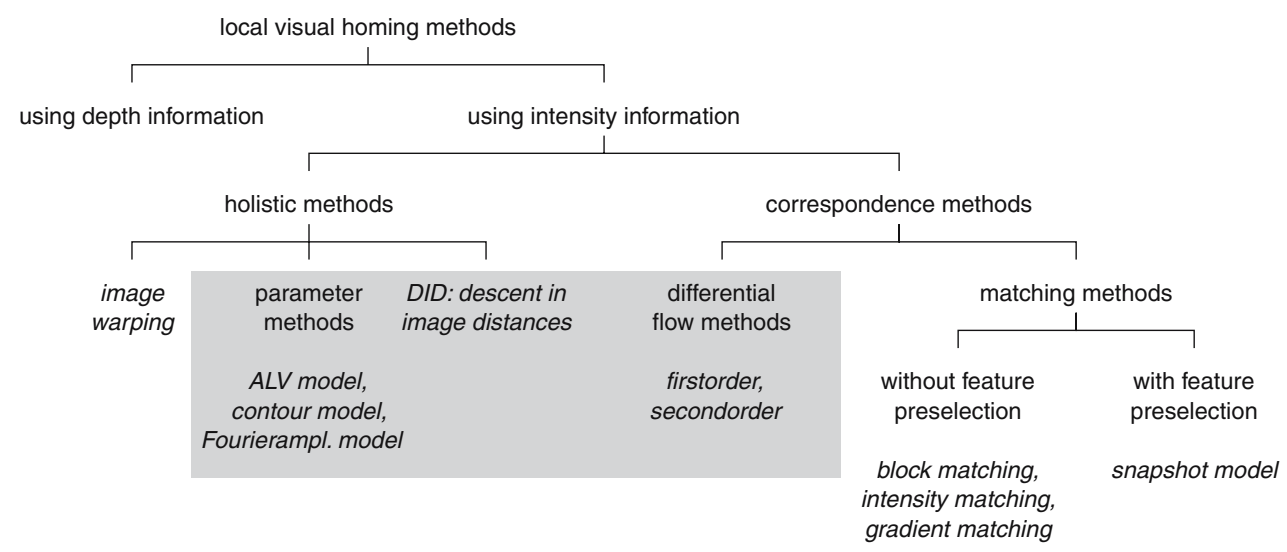

Fig. 1 Local visual homing methods. In this paper, we extend parameter methods and the DID method by flow templates and establish a relation with differential flow methods

vectors gives a viable estimate for the home direction. In matching methods, corresponding regions are found by searching for the best-matching region in the neighborhood. The search can be restricted to preselected features (e.g., edges), or each local region can be considered without any preselection. The algorithmic snapshot model suggested by Cartwright and Collett (1983) is the classical representative of a matching method with feature preselection; in this case, the features are dark and bright regions.

Matching methods without feature preselection have their roots in methods for the computation of optical flow (we refer to these methods as "flow-based matching methods" below). In block matching, the matching is based on the pixel-by-pixel distance between two rectangular regions in two images, in intensity matching, the block is reduced to a single pixel, and gradient matching searches for the best-matching gradient (Vardy and Möller 2005). Differential flow methods are derived from Taylor approximations of correspondence equations between intensities (first-order) or between gradients (second-order) under the assumption of small shifts (Barron et al. 1994). Vardy and Möller (2005) reported that flow-based matching methods and differential flow methods yield surprisingly robust navigation behavior. This is especially surprising for differential flow methods, since the above-mentioned assumption of small shifts is violated for the long spatial distance between the target and the current location. Also in the matching methods the search was restricted to the vicinity of a region; thus some regions will have moved beyond the search range. Vardy and Möller (2005) explain this good performance by the fact that, even though the flow fields are of relatively low quality, there is still a sufficiently large number of flow vectors, particularly in the vicinity of the foci of expansion and contraction, that fulfill the assumption of small shifts and therefore produce a concordant vote for the direction of the home vector, whereas the erroneous flow vectors yield uncorrelated movement directions and are therefore overruled.

Holistic methods, rather than trying to find correspondences between local regions in two images, treat an image as a whole. The warping method proposed by Franz et al. (1998b) is essentially a process of "mentally" simulating how different movements would distort (warp) the current image. By searching through the space of movement parameters for that distorted image which best fits the stored snapshot, the method produces estimates for the home direction, the orientation of the agent, and the distance from home. The search is based on the assumption that all landmarks are found in roughly the same distance from the agent. Due to the large search space, the warping method is only practically feasible if the image is reduced to a horizontal panoramic view of only one pixel height (see also Sect. 5.4). Despite these restrictions, the warping method produces very reliable homing behavior in different environments; it is only outperformed by some flow-based matching methods and differential flow methods that, however, require a compass to align the two images (Vardy and Möller 2005).

A subclass of holistic methods is embraced by the term parameter methods. They are based on the assumption that it is sufficient to only store a condensed description of the snapshot image, a so-called "parameter vector." Homing is then accomplished by some optimization method, often a simple gradient descent, applied on a distance measure between the parameter vectors obtained from the image at the target location and at the current location. Parameter methods have been suggested as a possible explanation for peculiar 
observations in experiments with desert ants (Möller 2001) and bees (Möller 2000). A special instance of parameter models is the average landmark vector $(A L V)$ model (Lambrinos et al. 2000), where the parameter vector comprises the two components of the average of unit vectors pointing to selected landmark features. While usually in parameter methods the distance measure or "potential" can be computed but the home vector has to be estimated by sampling the potential at multiple locations in space, the ALV method directly provides a home vector (it is equal to the difference between the two average landmark vectors), whereas the potential cannot be computed because it depends on the unknown distances to the landmarks (Möller 2002). While these parameter methods are of mostly theoretical interest, the Fourier-amplitude method suggested by Menegatti et al. (2004) may indeed be suitable for realworld applications. In this method, a panoramic image (with cyclic rows) is characterized by the first few Fourier amplitudes of each row. Since Fourier amplitudes are invariant against shifts, the parameter vector has the desirable property of being invariant against rotations of the agent, and the method can thus be used without a compass. In a recent publication, Stürzl and Mallot (2006) derived another Fourier-based method from the warping method.

Another promising holistic method is the descent in image distances (DID) introduced by Zeil et al. (2003). The method was derived from the observation that, in natural images, the root mean squared error (RMS error) between snapshot and current view smoothly rises with increasing spatial distance between current and target location (see gray tones in Fig. 2). Thus, similar to parameter models, the target location can be reached by applying a movement strategy derived from some optimization method on the distance measure (here the RMS error). This can be a form of the Gauss-Seidel strategy (move straight while the distance measure decreases; otherwise turn $\pm 90^{\circ}$ ) as in the "RunDown" strategy used by Zeil et al. (2003), or the gradient of the distance measure can be estimated repeatedly. For the latter, the agent has to collect at least three samples of the distance measure on noncollinear locations; usually, the three sampling points form a right triangle. The negative gradient estimated from the potential at these three points is the home vector (see vectors in Fig. 2).

In a practical application, however, these movement strategies are not desirable, since they require sharp turns of the robot and increase the overall length of the homeward journey. Especially in applications like cleaning where the robot has to follow a given trajectory, test steps are impractical. Moreover, estimates of the gradient are affected by odometry errors, since the relative position of the sampling points has to be known. In this paper, we suggest a novel method to determine an estimate of the gradient without the necessity of inserting test steps to sample the distance measure. This concept is generally applicable to all homing methods that can be formulated as a gradient descent or ascent in a distance measure. We focus on applying the idea to the DID method since this method is promising for real-world applications and distinguishes itself by its simplicity and low computational complexity. In the discussion we demonstrate the broader applicability by outlining three alternative methods derived from the same framework. Being able to save the test steps and instead compute the home vector directly would make a whole class of methods more attractive for applications.

The core idea of our approach is to predict how the image would change under two small, mutually perpendicular movements and to estimate the gradient from the distance measure applied to the current image and the two predicted images. The image prediction is accomplished by projecting the intensity gradient onto two fixed template flow fields or "matched filters" for purely translational movements. These flow fields have a typical form: a focus of expansion in the direction of movement, a focus of contraction in the opposite direction, and regions with approximately horizontal flow between them (Fig. 4). The direction of the flow vectors in the two templates does not depend on the distance to the objects in the world, but distance only affects the length of these vectors. Since depth information is not available, we can only assume that all objects are located in approximately the same distance from the current position. The matched-filter method directly delivers the home vector by a pixelwise summation; both the image prediction and the computation of the distance measure are performed implicitly.

Starting from a description that covers both parameter models and the DID method, we derive the matchedfilter DID method (MFDID) in Sect. 2. It turns out that MFDID exhibits a structural resemblance to first-order differential flow methods, as tested by Vardy and Möller (2005); this relation is analyzed in Sect. 3. The performance of the MFDID method is compared with the original DID method and with a first-order differential flow method by applying it to indoor image databases in Sect. 4. We discuss the experimental results and alternative matched-filter methods in Sect. 5.

\section{Derivation of matched-filter DID}

Notation Let $\boldsymbol{\varphi}_{i j}=\left(\beta_{i}, \gamma_{j}\right)^{\mathrm{T}}$ be a $2 \mathrm{D}$ vector of angles in spherical coordinates describing the direction from 
Fig. 2 DID method applied to indoor image database (lab environment, see Sect. 4). Gray tones in each square correspond to the RMS error between the image taken at the position in the center of the square and the snapshot image taken in the center of the white square (white: maximal value, black: zero). The home vectors are obtained by estimating the gradient from the RMS values at this and at two neighboring grid positions in perpendicular directions. Left: Home position (5,7). Right: Home position $(5,16)$
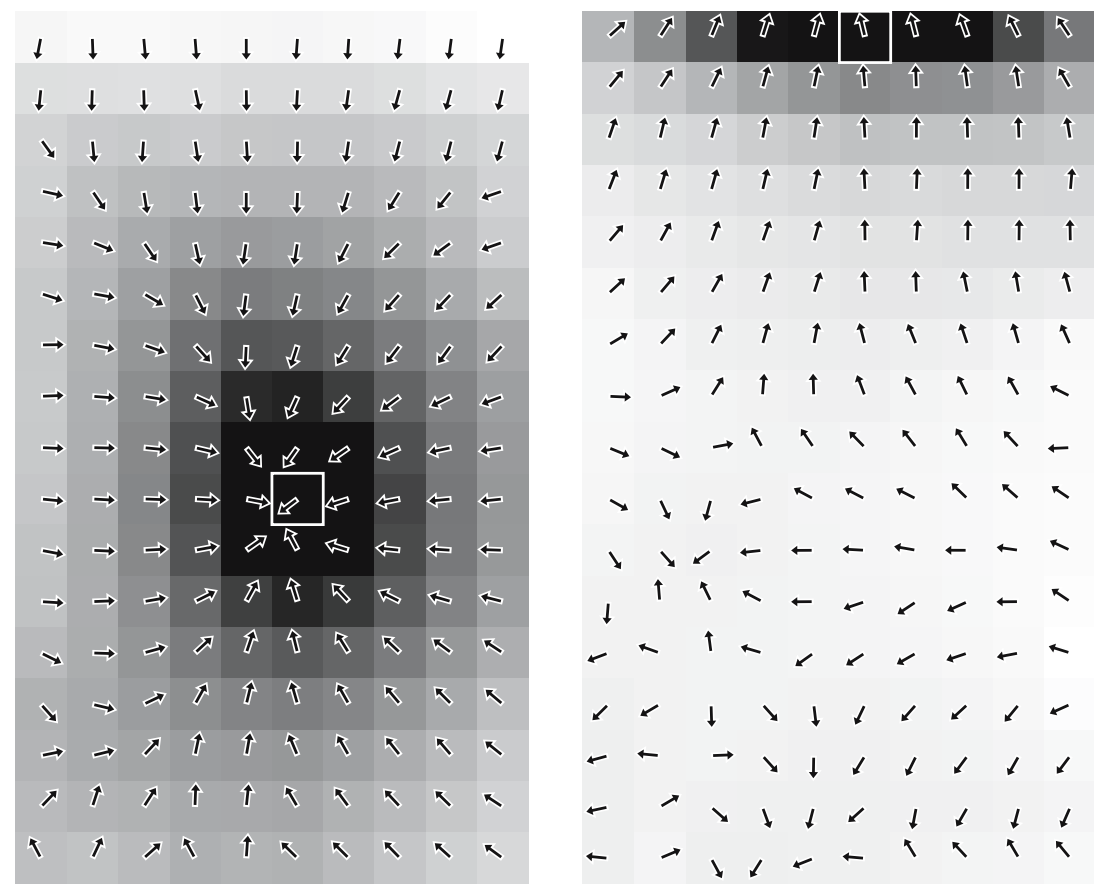

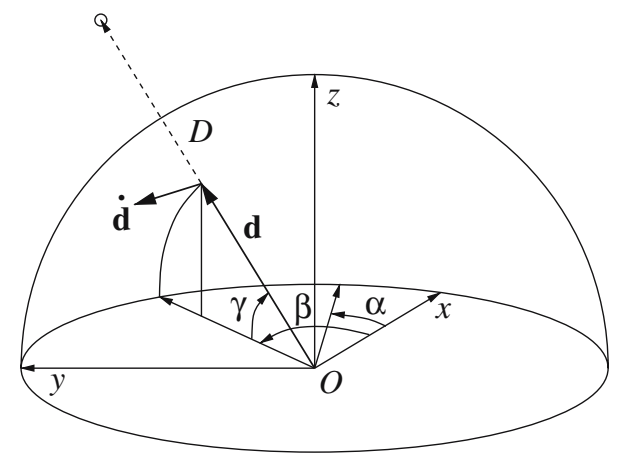

Fig. 3 In spherical coordinates, the unit vector d pointing toward a feature of distance $D$ from the origin $O$ is expressed by a horizontal angle $\beta$ and a vertical angle $\gamma$. When the agent moves in direction $\alpha$ in the $x-y$ plane, the feature shifts in direction $\dot{\mathbf{d}}$

the vantage point of the camera to the visual feature in the environment that appears at pixel coordinates $(i, j)^{\mathrm{T}}$ in the image. The horizontal angle $\beta_{i}$ varies only with pixel coordinate $i$, the vertical angle $\gamma_{j}$ only with $j$ $\left(\gamma_{j}=0\right.$ corresponds to the horizon, positive $\gamma_{j}$ are above the horizon). In the horizontal direction, the image is closed to a panoramic view. We will omit the pixel index in some steps of the derivation. Figure 3 visualizes the geometrical relations.

Also, let $\mathbf{x}=(x, y)^{\mathrm{T}}$ be the position of the vantage point in the horizontal plane. We assume that the camera coordinate system is aligned with a fixed world coordinate system by using some sort of compass. With $C\left(\boldsymbol{\varphi}_{i j}, \mathbf{x}\right)$ we describe a pixel $(i, j)^{\mathrm{T}}$ in the image taken at position $\mathbf{x}$. In local visual homing, a snapshot image is captured at the goal location $\mathbf{x}_{0}$ and stored, denoted henceforth as $S\left(\varphi_{i j}\right)=C\left(\varphi_{i j}, \mathbf{x}_{0}\right)$. If we refer to pixel coordinates, we use the abbreviations $C_{i j}(\mathbf{x})=C\left(\boldsymbol{\varphi}_{i j}, \mathbf{x}\right)$ and $S_{i j}=S\left(\varphi_{i j}\right)$. Entire images are represented by $C(\mathbf{x})$ and $S=C\left(\mathbf{x}_{0}\right)$. All images are assumed to be monochrome.

Descent in distance measures In a general formulation covering both parameter methods and the DID method, a distance measure (potential) $p(\mathbf{x})$ is defined over the plane (coordinate $\mathbf{x}$ ):

$p(\mathbf{x})=P\{f[C(\mathbf{x})], f[S]\}$.

The home vector is the negative spatial gradient of the potential:

$\mathbf{h}(\mathbf{x})=-\nabla_{\mathbf{x}} p(\mathbf{x})$.

The potential is determined by applying a distance measure expressed by $P$ to a feature vector derived from the current view $C(\mathbf{x})$ and a feature vector derived from the snapshot $S$. The transformation from images to feature vectors is described by function $f$. In the ALV model, for example, $f$ would produce the two components of the average landmark vector (Lambrinos et al. 2000); in the contour model, it would amount to a computation of contour length and contour eccentricity (Möller 2001); in the Fourier-amplitude model, $f$ would deliver the first few Fourier amplitudes for each image row (Menegatti et al. 2004).

The DID model is a special case where $f$ is the identity mapping:

$p(\mathbf{x})=P\{C(\mathbf{x}), S\}$. 
More specifically, Zeil et al. (2003) use the root mean squared error (RMS) error as distance measure

$$
P\{C, S\}=\operatorname{RMS}\{C, S\}=\sqrt{\frac{1}{N} \sum_{i, j}\left[C_{i j}-S_{i j}\right]^{2}},
$$

where $N$ is the number of pixels included in the measure. This allows a comparison of image distance values if different parts of the image are excluded from the analysis; Zeil et al. (2003) used this to remove parts of the camera gantry visible in their images.

SSE gradient In the MFDID method, we use the sum squared error (SSE) instead of the RMS (root of SSE normalized to number of pixels) suggested by Zeil et al. (2003). This modification has no effect on the direction of the gradient but is more amenable to analytical treatment. The SSE between the current image $C\left(\varphi_{i j}, \mathbf{x}\right)$ and the snapshot $S\left(\varphi_{i j}\right)$ is defined as

$p(\mathbf{x})=\operatorname{SSE}(\mathbf{x})=\frac{1}{2} \sum_{i, j}\left[C\left(\boldsymbol{\varphi}_{i j}, \mathbf{x}\right)-S\left(\boldsymbol{\varphi}_{i j}\right)\right]^{2}$.

The home vector $\mathbf{h}(\mathbf{x})$ is the negative gradient of the SSE:

$$
\begin{aligned}
\mathbf{h}(\mathbf{x}) & =-\nabla_{\mathbf{x}} \operatorname{SSE}(\mathbf{x}) \\
& =-\sum_{i, j} \nabla_{\mathbf{x}} C\left(\varphi_{i j}, \mathbf{x}\right) \cdot\left[C\left(\varphi_{i j}, \mathbf{x}\right)-S\left(\varphi_{i j}\right)\right] .
\end{aligned}
$$

Spatial gradient and intensity gradient For small test steps $\Delta \mathbf{x}$, the spatial gradient $\nabla_{\mathbf{x}} C\left(\varphi_{i j}, \mathbf{x}\right)$ can be related to the intensity gradient in the following way. A small movement in direction $\Delta \mathbf{x}$ will cause a small shift of the feature at $\varphi$ in the image by $\Delta \varphi=\Delta \varphi(\varphi, \mathbf{x}, \Delta \mathbf{x})$. This shift depends on the movement $(\Delta \mathbf{x})$ and on the distance to the feature in the environment (and thus on $\mathbf{x})$. By Taylor expansion of $C(\boldsymbol{\varphi}+\Delta \boldsymbol{\varphi}, \mathbf{x}+\Delta \mathbf{x})$ for both arguments at the point $(\varphi, \mathbf{x})$ we obtain

$$
\begin{aligned}
& C(\boldsymbol{\varphi}+\Delta \boldsymbol{\varphi}, \mathbf{x}+\Delta \mathbf{x}) \\
& \quad \approx C(\boldsymbol{\varphi}, \mathbf{x})+\nabla_{\varphi}^{\mathrm{T}} C(\boldsymbol{\varphi}, \mathbf{x}) \cdot \Delta \varphi+\nabla_{\mathbf{x}}^{\mathrm{T}} C(\boldsymbol{\varphi}, \mathbf{x}) \cdot \Delta \mathbf{x} .
\end{aligned}
$$

If we assume that the intensity of the pixel does not change markedly when the pixel is shifted, i.e.,

$C(\boldsymbol{\varphi}+\Delta \boldsymbol{\varphi}, \mathbf{x}+\Delta \mathbf{x}) \approx C(\boldsymbol{\varphi}, \mathbf{x})$,

then we find that

$-\nabla_{\varphi}^{\mathrm{T}} C(\varphi, \mathbf{x}) \cdot \Delta \varphi \approx \nabla_{\mathbf{x}}^{\mathrm{T}} C(\varphi, \mathbf{x}) \cdot \Delta \mathbf{x}$,

which establishes the relation between the spatial gradient $\nabla_{\mathbf{x}} C(\varphi, \mathbf{x})$ and $\nabla_{\varphi} C(\varphi, \mathbf{x})$, the intensity gradient of the current view.
Optical flow For small movements, the shift $\Delta \varphi$ can be determined from the flow equation derived by Koenderink and van Doorn (1987): When the coordinate system of the camera moves with speed $\dot{\mathbf{x}}$ and performs a rotation around $\omega$ with speed $\|\omega\|$, the flow vector $\dot{\mathbf{d}}$ that describes the visual movement of a feature in direction $\mathbf{d}$ (with $\|\mathbf{d}\|=1$ ) and distance $D$ from the vantage point is obtained from

$\dot{\mathbf{d}}=-\frac{\dot{\mathbf{x}}-\left(\dot{\mathbf{x}}^{\mathrm{T}} \mathbf{d}\right) \mathbf{d}}{D}-\boldsymbol{\omega} \times \mathbf{d}$.

In our case, the movement is restricted to pure translation, thus $\boldsymbol{\omega}=\mathbf{0}$. For a movement in the $x-y$ plane in direction $\alpha$ and with speed $v$

$\dot{\mathbf{x}}=(\dot{x}, \dot{y}, 0)^{\mathrm{T}}=v(\cos \alpha, \sin \alpha, 0)^{\mathrm{T}}$,

and with $\mathbf{d}$ and $\dot{\mathbf{d}}$ expressed in spherical coordinates as $\boldsymbol{\varphi}=(\beta, \gamma)$ and $\dot{\boldsymbol{\varphi}}=(\dot{\beta}, \dot{\gamma})^{\mathrm{T}}$, respectively, we obtain

$$
\begin{aligned}
\left(\begin{array}{l}
\dot{\beta} \\
\dot{\gamma}
\end{array}\right) & =\frac{v}{D} \underbrace{\left(\begin{array}{cc}
\sec \gamma & 0 \\
0 & \sin \gamma
\end{array}\right)}_{\boldsymbol{\Gamma}} \underbrace{\left(\begin{array}{cc}
\sin \beta & -\cos \beta \\
\cos \beta & \sin \beta
\end{array}\right)}_{\mathbf{B}}\left(\begin{array}{l}
\cos \alpha \\
\sin \alpha
\end{array}\right) \\
& =\frac{1}{D} \boldsymbol{\Gamma} \mathbf{B}\left(\begin{array}{l}
\dot{x} \\
\dot{y}
\end{array}\right),
\end{aligned}
$$

where $\sec \gamma=(\cos \gamma)^{-1}$. Equation (6) transforms a movement into a flow vector. For a small movement $\Delta \mathbf{x}=(\Delta x, \Delta y)^{\mathrm{T}}$ in the plane, we can approximate

$\Delta \varphi=\frac{1}{D} \Gamma \mathbf{B} \Delta \mathbf{x}$.

For movements in the direction of the coordinate axes $\mathbf{e}_{1}$ and $\mathbf{e}_{2}$ with small length $\eta \ll 1$ described by $\Delta \mathbf{x}_{1}=\eta \mathbf{e}_{1}$ and $\Delta \mathbf{x}_{2}=\eta \mathbf{e}_{2}$ we see from Eq. (7) that

$\Delta \boldsymbol{\varphi}_{1}=\frac{\eta}{D} \boldsymbol{\Gamma} \mathbf{B} \mathbf{e}_{1}, \quad \Delta \boldsymbol{\varphi}_{2}=\frac{\eta}{D} \boldsymbol{\Gamma} \mathbf{B} \mathbf{e}_{2}$.

These equations describe the two flow templates (matched filters) shown in Fig. 4. $\Delta \varphi_{1}$ is the flow field experienced under the movement $\Delta \mathbf{x}_{1}$ along the $x$ direction, and $\Delta \varphi_{2}$ is the flow field for the movement $\Delta \mathbf{x}_{2}$ along the $y$ direction. When these flow fields and movements are inserted into Eq. (4), we see that each component of the spatial gradient is related to one of the two flow fields.

Home vector We now insert Eq. (7) into Eq. (4). For arbitrary $\Delta \mathbf{x}$, we obtain the following expression for the spatial gradient:

$\nabla_{\mathbf{x}} C(\boldsymbol{\varphi}, \mathbf{x}) \approx-\frac{1}{D} \mathbf{B}^{\mathrm{T}} \boldsymbol{\Gamma} \nabla_{\varphi} C(\boldsymbol{\varphi}, \mathbf{x})$.

Here, $D=D(\varphi)$ is the distance of the feature at angular coordinate $\varphi$. Since we have no information about object 

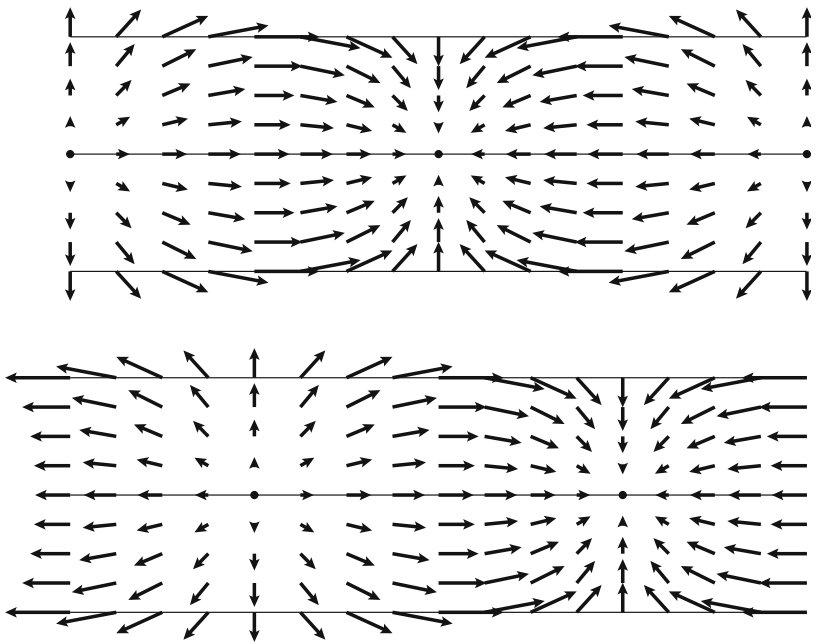

Fig. 4 Flow templates for translations in direction $\mathbf{e}_{1}$ (top) and $\mathbf{e}_{2}$ (bottom) obtained from Eq. (8). The horizontal axis is $\beta$ $(0 \ldots 2 \pi)$, the vertical axis $\gamma(-1 \ldots 1)$. Lines: horizon $(\gamma=0)$ and maximal/minimal value of $\gamma$ as well as range of $\beta$. Dots: foci of expansion and contraction. The flow vectors are scaled for good visibility

distances, we continue with an equal-distance assumption (see, e.g., Franz et al. 1998b): $D$ is assumed to be constant for all directions $\varphi$. We finally get an equation for the home vector in Eq. (3)

$$
\begin{aligned}
\mathbf{h}(\mathbf{x})= & \sum_{i, j} \frac{1}{D} \mathbf{B}\left(\beta_{i}\right)^{\mathrm{T}} \boldsymbol{\Gamma}\left(\gamma_{j}\right) \cdot \nabla_{\varphi} C\left(\boldsymbol{\varphi}_{i j}, \mathbf{x}\right) \\
& \times\left[C\left(\boldsymbol{\varphi}_{i j}, \mathbf{x}\right)-S\left(\boldsymbol{\varphi}_{i j}\right)\right] .
\end{aligned}
$$

Image processing For the derivation above, we used angular coordinates $\boldsymbol{\varphi}_{i j}=\left(\beta_{i}, \gamma_{j}\right)^{\mathrm{T}}$; if Eq. (10) is implemented in an image processing system, however, we have to switch to pixel coordinates $(i, j)^{\mathrm{T}}$ to express $\nabla_{\varphi} C\left(\varphi_{i j}, \mathbf{x}\right)$ by standard filter operations. For simplicity we assume that the image has been preprocessed in such a way that we have an approximately linear relationship between $\beta_{i}$ and $i$ and between $\gamma_{j}$ and $j$, like

$\beta_{i}=2 \pi-\frac{2 \pi i}{w}, \quad \gamma_{j}=\gamma_{0}+\frac{\left(\gamma_{h-1}-\gamma_{0}\right) j}{h-1}$

for an image of width $w$ and height $h$ pixels, where $i$ decreases with increasing $\beta_{i}$ and $j$ decreases with increasing $\gamma_{j}$. The bottom and top rows correspond to $\gamma_{h-1}$ and $\gamma_{0}$, respectively. For equal angle-to-pixel ratios $\delta$ in vertical and horizontal directions

$\delta=\frac{2 \pi}{w}=\frac{\gamma_{0}-\gamma_{h-1}}{h-1}$ we get $\beta_{i}=2 \pi-\delta i$ and $\gamma_{j}=\gamma_{0}-\delta j$. We can then express the gradient in pixel coordinates by

$$
\begin{aligned}
\nabla_{i j} C_{i j}(\mathbf{x}) & =\left(\begin{array}{c}
\frac{\partial}{\partial i} C_{i j}(\mathbf{x}) \\
\frac{\partial}{\partial j} C_{i j}(\mathbf{x})
\end{array}\right)=\left(\begin{array}{c}
\frac{\partial}{\partial \beta} C\left(\boldsymbol{\varphi}_{i j}, \mathbf{x}\right) \frac{\partial \beta}{\partial i} \\
\frac{\partial}{\partial \gamma} C\left(\boldsymbol{\varphi}_{i j}, \mathbf{x}\right) \frac{\partial \gamma}{\partial j}
\end{array}\right) \\
& =-\delta \nabla_{\varphi} C\left(\boldsymbol{\varphi}_{i j}, \mathbf{x}\right) .
\end{aligned}
$$

$\nabla_{i j} C_{i j}(\mathbf{x})$ can easily be determined from a discrete approximation by applying simple filter kernels like $\frac{1}{2}(-1,0,1)$ and $\frac{1}{2}(-1,0,1)^{\mathrm{T}}$ to the pixels of the current image. Since $D$ in Eq. (10), $\delta$ in Eq. (11), and the factor $\frac{1}{2}$ in the kernels are positive constants and just affect the length of the home vector, we can omit these factors from the computation of Eq. (10) and determine the home vector from

$\tilde{\mathbf{h}}(\mathbf{x})=\sum_{i, j} \underbrace{\mathbf{B}\left(\beta_{i}\right)^{\mathrm{T}} \boldsymbol{\Gamma}\left(\gamma_{j}\right)}_{\mathbf{T}_{i j}} \cdot \nabla_{i j} C_{i j}(\mathbf{x}) \cdot\left[S_{i j}-C_{i j}(\mathbf{x})\right]$,

where the matrices $\mathbf{T}_{i j}$ can be precomputed. Note that the negative sign in Eq. (11) was incorporated into the last factor. The tilde signifies that this home vector is computed from an intensity gradient related to pixel coordinates rather than angular coordinates and that we omitted the factors as described above; this is the home vector computation used in our implementation.

\section{Analysis of matched-filter DID}

In what follows, we investigate the relation between the MFDID method in Eq. (10) and the flow vectors computed by a first-order differential method applied to the snapshot and the current view.

Flow-based navigation Vardy and Möller (2005) applied first-order differential flow methods to the problem of visual homing. In this application, the flow vector field is determined between the snapshot image $S(\varphi)=$ $C\left(\boldsymbol{\varphi}, \mathbf{x}_{0}\right)$ and the current view $C(\boldsymbol{\varphi}, \mathbf{x})$ with $\mathbf{x}=\mathbf{x}_{0}+\Delta \mathbf{x}$. First-order methods rest on the assumption that the image intensity is unchanged under a shift $\Delta \varphi$ (Barron et al. 1994):

$C(\varphi+\Delta \varphi, \mathbf{x})=S(\varphi)$.

By Taylor expansion of the 1.h.s. for its first argument and approximation to the first order, we get

$C(\boldsymbol{\varphi}, \mathbf{x})+\nabla_{\varphi}^{\mathrm{T}} C(\boldsymbol{\varphi}, \mathbf{x}) \Delta \varphi=S(\varphi)$.

The solution to this flow equation is not unique due to the aperture problem. Choosing the flow vector $\Delta \varphi$ parallel to the intensity gradient, we gain

$\Delta \varphi=\frac{\nabla_{\varphi} C(\boldsymbol{\varphi}, \mathbf{x})}{\left\|\nabla_{\varphi} C(\boldsymbol{\varphi}, \mathbf{x})\right\|^{2}} \cdot[S(\boldsymbol{\varphi})-C(\boldsymbol{\varphi}, \mathbf{x})]$ 
as one possible solution that fulfills Eq. (13) (see, e.g., Beauchemin and Barron (1995)). The flow vector $\Delta \varphi$ at the pixel in direction $\varphi$ describes the shift of a feature from its position in the snapshot to its position in the current view. By a "vector mapping" method such as the one described by Vardy and Möller (2005) or by inverting Eq. (7), the flow vector can be transformed into a corresponding direction of movement.

MFDID versus differentialflow The structural resemblance between Eq. (14) and the summands in Eq. (10) is apparent: The summands are proportional to the flow vector obtained from applying a first-order differential flow method to the snapshot and the current view. Neglecting the division by the squared length of the intensity gradient, we can therefore interpret the summands of Eq. (10) as flow vectors that are transformed into the corresponding direction of movement in the plane; the latter could be called a "local home vector"

$$
\begin{aligned}
\mathbf{h}_{i j}(\mathbf{x})= & \frac{1}{D} \mathbf{B}\left(\beta_{i}\right)^{\mathrm{T}} \boldsymbol{\Gamma}\left(\gamma_{j}\right) \cdot \nabla_{\varphi} C\left(\boldsymbol{\varphi}_{i j}, \mathbf{x}\right) \\
& \times\left[C\left(\boldsymbol{\varphi}_{i j}, \mathbf{x}\right)-S\left(\boldsymbol{\varphi}_{i j}\right)\right],
\end{aligned}
$$

with the overall home vector being determined from summing over $i$ and $j$. In what follows, we establish a relationship between the flow vector obtained from Eq. (14), referred to as $\Delta \varphi_{i j}^{*}$, and the flow vector $\Delta \varphi_{i j}$ corresponding to the local home vector of the MFDID method in Eq. (15). By inserting $\Delta \varphi_{i j}^{*}$ from Eq. (14) into Eq. (15), we get

$$
\mathbf{h}_{i j}(\mathbf{x})=-\frac{1}{D} \mathbf{B}\left(\beta_{i}\right)^{\mathrm{T}} \boldsymbol{\Gamma}\left(\gamma_{j}\right) \cdot\left\|\nabla_{\varphi} C\left(\boldsymbol{\varphi}_{i j}, \mathbf{x}\right)\right\|^{2} \cdot \Delta \boldsymbol{\varphi}_{i j}^{*} .
$$

Here, $D$ is the average distance to the landmarks that is used in the prediction steps. In contrast to the flow fields obtained from the small test steps in the MFDID method, the flow vectors relating the current view to the snapshot result from usually long spatial distances. This violates the assumptions underlying the derivation of Eq. (13); nevertheless, differential flow methods seem to produce relatively good homing performance (Vardy and Möller 2005). For our analysis, we assume that the spatial distance between the snapshot and the current view is small, and can therefore transform $\mathbf{h}_{i j}(\mathbf{x})$ into the corresponding flow vector as $\Delta \mathbf{x}$ according to Eq. (7):

$$
\begin{aligned}
\Delta \boldsymbol{\varphi}_{i j}= & \frac{1}{D_{i j}} \boldsymbol{\Gamma}\left(\gamma_{j}\right) \mathbf{B}\left(\beta_{i}\right) \mathbf{h}_{i j}(\mathbf{x}) \\
= & -\frac{1}{D_{i j} D} \boldsymbol{\Gamma}\left(\gamma_{j}\right) \mathbf{B}\left(\beta_{i}\right) \mathbf{B}\left(\beta_{i}\right)^{\mathrm{T}} \boldsymbol{\Gamma}\left(\gamma_{j}\right) \\
& \times\left\|\nabla_{\varphi} C\left(\boldsymbol{\varphi}_{i j}, \mathbf{x}\right)\right\|^{2} \cdot \Delta \boldsymbol{\varphi}_{i j}^{*} .
\end{aligned}
$$

$D_{i j}$ is the true distance to the feature in the world. Since $\mathbf{B B}^{\mathrm{T}}=\mathbf{I}$, we end with the relation

$$
\begin{aligned}
\Delta \boldsymbol{\varphi}_{i j}= & -\frac{1}{D_{i j} D}\left(\begin{array}{cc}
\sec ^{2} \gamma_{j} & 0 \\
0 & \sin ^{2} \gamma_{j}
\end{array}\right) \\
& \times\left\|\nabla_{\varphi} C\left(\boldsymbol{\varphi}_{i j}, \mathbf{x}\right)\right\|^{2} \cdot \Delta \boldsymbol{\varphi}_{i j}^{*} .
\end{aligned}
$$

This is a relation between two flow vectors. On the 1.h.s. we find the flow vector $\Delta \varphi_{i j}$ that we would obtain if the agent moved along the local home vector $\mathbf{h}_{i j}(\mathbf{x})$ produced by the MFDID method. The r.h.s. contains the flow vector $\Delta \varphi_{i j}^{*}$ at the same position in the image that would be produced by a first-order differential flow method when it is applied to the snapshot and the current view.

The negative sign derives from the fact that the local home vector of the MFDID method describes a movement toward the target location whereas the flow vector determined by the differential method describes a movement from the target location to the current location. Furthermore, the relation includes the squared ratio between the length of the gradient and the feature distance, which can be explained as a property of the DID method. The DID method establishes a relation between the spatial distance and the image distance. This relation depends on the distance to the feature (the larger the distance of an object, the smaller that object's impact on the image distance) and on the intensity gradient (the larger the intensity gradient, the larger its influence on the image distance). Finally, the matrix multiplies the horizontal component of $\Delta \varphi_{i j}^{*}$ by $\sec ^{2} \gamma_{j}$ and the vertical component with $\sin ^{2} \gamma_{j}$. For the usually small vertical range of the images, the first multiplication should have a negligible effect. The second multiplication reveals that the MFDID method (1.h.s.) seems to underestimate the vertical components of flow vectors close to the horizon $(\gamma=0)$ relative to the first-order differential flow method.

\section{Experiments}

\subsection{Image database}

The database of images collected by Vardy and Möller (2005) is used for all experiments described below. This database is publicly available at www . ti . uni-bielefeld.de/html/research/avardy. Images from this database were captured by a mobile robot equipped with a panoramic imaging system. This imaging system consists of a camera pointed upward at a hyperbolic mirror. Images from two different environments are used here. The first environment is a computer lab of dimen- 
sions $5.5 \times 8.25 \mathrm{~m}$. Within this lab the capture area was $2.7 \times 4.8 \mathrm{~m}$ with images captured on a grid with $30-\mathrm{cm}$ resolution. The second environment is the main hall of Bielefeld University, a large open space where the distance of viewed objects ranges from several meters to hundreds of meters. The capture grid in the hall had dimensions $4.5 \times 10 \mathrm{~m}$ at 50 -cm resolution. ${ }^{1}$

All images were captured at approximately the same orientation. If the methods described here were to be used for the online control of a mobile robot, some kind of compass would have to be employed. Since magnetic compasses tend to be problematic in indoor environments, orientation could be derived from visual information; the minimization of image distances over rotation can be used for this purpose as well (Zeil et al. 2003).

Raw images from the database were low-pass filtered using a Butterworth filter. The filtered images were then reprojected onto a sphere and unfolded to rectangular images of size $300 \times 50$. Rows in these images correspond to equal vertical angles above and below the horizon (center row); columns correspond to equal horizontal angles. The filter parameters were obtained by testing all methods using a variety of parameters and choosing a parameter set that worked well for all methods. The chosen parameters (relative cutoff frequency 0.01, order 3) yield strongly blurred images, an example of which can be seen in Fig. 5 (bottom). We discuss the impact of low-pass filtering in Sect. 5.1.

\subsection{Methods}

We compare three different methods [again, the tilde signifies home vectors as they are computed in our implementation, see comment below Eq. (12)]:

1. DID: The original DID method uses a current view and two nearby views in the database to estimate the negative gradient:

$$
\tilde{\mathbf{h}}_{1}(\mathbf{x})=-\left(\begin{array}{l}
\operatorname{SSE}\left(\left[x_{m+s}, y_{n}\right]^{\mathrm{T}}\right)-\operatorname{SSE}\left(\left[x_{m}, y_{n}\right]^{\mathrm{T}}\right) \\
\operatorname{SSE}\left(\left[x_{m}, y_{n+s}\right]^{\mathrm{T}}\right)-\operatorname{SSE}\left(\left[x_{m}, y_{n}\right]^{\mathrm{T}}\right)
\end{array}\right) .
$$

Here, $m$ and $n$ relate to the index of the database view on the grid, and $s$ is a step length, also in grid units (if not stated otherwise, we use $s=1$; thus views are taken from adjacent grid points). Increasing indices $m$ and $n$ correspond to increasing coordinates on the corresponding axis. When the indices

\footnotetext{
1 Images from the "lab environment" come from the original image collection of the database. Images from the "hall environment" come from image collection hal11.
}

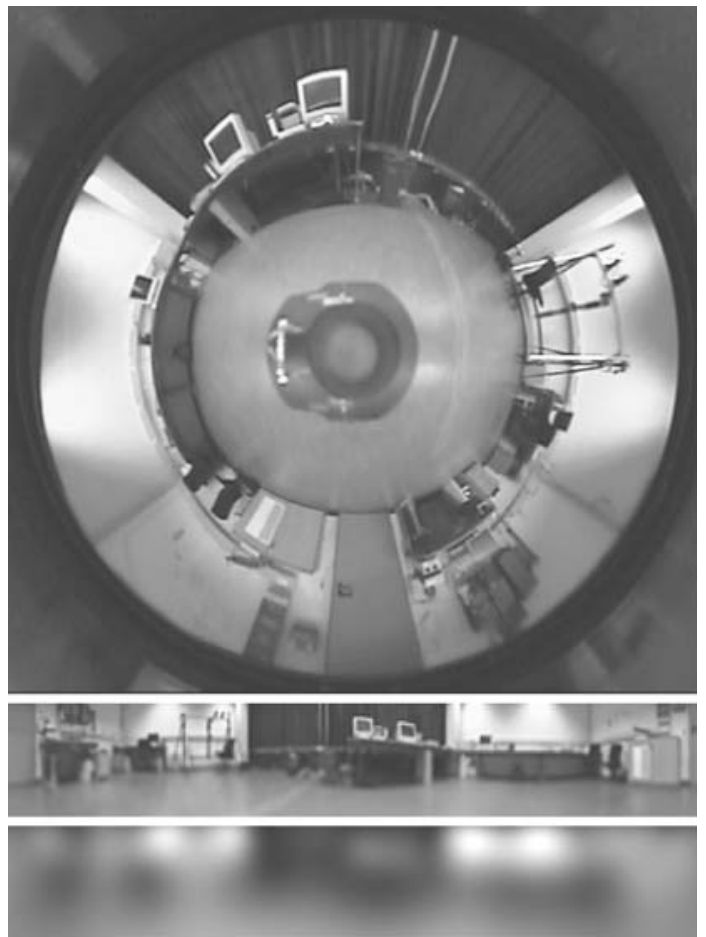

Fig. 5 Top: Image $(5,7)$ from lab. Center: The same image filtered at relative cutoff frequency 0.1 and unfolded. Bottom: Image filtered at cutoff frequency 0.01

$m+s$ or $n+s$ lie outside of the grid, $\left[x_{m-s}, y_{n}\right]^{\mathrm{T}}$ and $\left[x_{m}, y_{n-s}\right]^{\mathrm{T}}$ are taken instead and the sign of the corresponding component is inverted.

2. Matched-filter DID (MFDID): In the matched-filter version of the DID method, the home vector is computed from Eq. (12), given here again:

$$
\begin{aligned}
\tilde{\mathbf{h}}_{2}(\mathbf{x})= & \sum_{i, j} \mathbf{B}\left(\beta_{i}\right)^{\mathrm{T}} \boldsymbol{\Gamma}\left(\gamma_{j}\right) \\
& \times \nabla_{i j} C_{i j}(\mathbf{x}) \cdot\left[S_{i j}-C_{i j}(\mathbf{x})\right] .
\end{aligned}
$$

3. First-order differential flow (FirstOrder): The flow vector computed from the first-order differential flow method in Eq. (14) is inserted into the inverse of Eq. (7); if we assume that all visible points are at the same distance $D$, we can omit $D$ and obtain

$$
\begin{aligned}
\tilde{\mathbf{h}}_{3}(\mathbf{x})= & \sum_{i, j} \mathbf{B}\left(\beta_{i}\right)^{\mathrm{T}} \boldsymbol{\Gamma}\left(\gamma_{j}\right)^{-1} \\
& \times \frac{\nabla_{i j} C_{i j}(\mathbf{x})}{\left\|\nabla_{i j} C_{i j}(\mathbf{x})\right\|^{2}} \cdot\left[S_{i j}-C_{i j}(\mathbf{x})\right],
\end{aligned}
$$

where we applied $\mathbf{B}^{-1}=\mathbf{B}^{\mathrm{T}}$. The division by the square of the intensity gradient requires some thresholding scheme to prevent division by near-zero values. We have found that omitting this division leads 
to an improvement in the algorithm's performance. Hence, we utilize the following flow expression:

$$
\begin{aligned}
\tilde{\mathbf{h}}_{4}(\mathbf{x})= & \sum_{i, j} \mathbf{B}\left(\beta_{i}\right)^{\mathrm{T}} \boldsymbol{\Gamma}\left(\gamma_{j}\right)^{-1} \\
& \times \nabla_{i j} C_{i j}(\mathbf{x}) \cdot\left[S_{i j}-C_{i j}(\mathbf{x})\right] .
\end{aligned}
$$

\subsection{Assessment}

Homing performance is assessed by selecting one position in the capture grid as the goal and then applying the homing method to all other positions to obtain a home vector for each. This home vector field is then characterized by two performance metrics, as used before by Vardy and Möller (2005).

The average angular error ( $\mathrm{AAE}$ ) is the average angular distance between the computed home vector $\hat{\mathbf{h}}$ and the true home vector $\mathbf{h}$ (the angular distance between these two unit vectors is computed as $\arccos \left(\hat{\mathbf{h}}^{\mathrm{T}} \mathbf{h}\right) \in$ $[0, \pi])$. Note that the AAE is a linear statistical measure, not a circular one (Batschelet 1981). The return ratio $(\mathrm{RR})$ is computed by placing an agent at each nongoal position and allowing it to move according to the home vector for that position. We use steps with a length of 0.5 grid units. If the agent reaches within one grid unit of the goal after a fixed number of steps, the attempt is considered successful (the maximal number of steps is chosen such that the agent could reach the lower right corner of the grid from the upper left corner on an $\mathrm{L}$-shaped course). $\mathrm{RR}$ is defined as the ratio of the number of successful attempts to the total number of attempts.

As we are interested in robust performance throughout an environment, we test each homing method using all capture grid positions in turn as the goal position and then average the results. Henceforth, $\mathrm{AAE}_{*}$ and $\mathrm{RR}_{*}$ will refer to the average AAE and RR quantities across all goal positions.

\subsection{Results}

Home vectors for all methods for goal positions $(5,7)$ and $(5,16)$ of the lab environment are shown in Fig. 6. Relatively little difference between the methods is apparent for goal position $(5,7)$, although the performance metrics shown above each vector field indicate that MFDID exhibits the most correct field. The situation for goal position $(5,16)$ is quite different. Here we see excellent performance by FirstOrder but relatively poor performance by DID and MFDID.
Figure 7 presents plots of AAE and RR across all goal positions of the lab environment. For FirstOrder, the performance is generally good throughout. However, for DID and MFDID, the performance is quite poor along the line $y=16$.

Figure 8 summarizes the results for the hall environment. Here it is MFDID that exhibits both the lowest $\mathrm{AAE}_{*}$ and the highest $\mathrm{RR}_{*}$ values. In particular, the $\mathrm{RR}$ plots show that this method is the most consistent in this environment.

Qualitatively, we find the performance of all methods tested above quite comparable. The method based on first-order differential flow performs better in the lab environment, where both DID and MFDID experience a problem in one small region of the capture grid. In the hall environment, MFDID clearly performs the best of all methods.

\section{Discussion}

\subsection{Analysis of the performance}

In this section we discuss several issues that are relevant to the performance of the methods studied in this paper.

First, we consider the issue of low-pass filtering. As shown in Fig. 5 (bottom), the filter parameters adopted yield very strong low-pass filtering. To assess the impact on performance, we tested all three methods by fixing the order of the Butterworth low-pass filter at 3 and varying the relative cutoff frequency. The results are shown in Fig. 9. Note that lower cutoff frequencies yield a stronger low-pass-filtering effect (i.e., more blurry images). The chosen cutoff frequency of 0.01 appears to be optimal for all methods in the range examined. For smaller cutoff frequencies the performance drops drastically for all methods. For cutoff frequencies higher than 0.01 , there is a slight reduction in performance for all methods, except for MFDID as measured by return ratio. Generally, it appears that all methods are relatively insensitive to the filter frequency, as long as it remains higher than 0.01 .

The performance of both DID and MFDID depends on the spatial structure of the image distance function. For the concept of gradient DID to be applicable, the 2D image distance function must rise smoothly and monotonically with spatial distance. Figure 2 (left) shows this image distance function for goal position $(5,7)$ in the lab environment. Overlaid on this figure are the home vectors generated by DID. This distance function exhibits the necessary properties: a smooth monotonic rise from the single global maximum. Figure 2 (right) shows the image distance function for goal position $(5,16)$. In this case, the distance function fails to exhibit the desired 


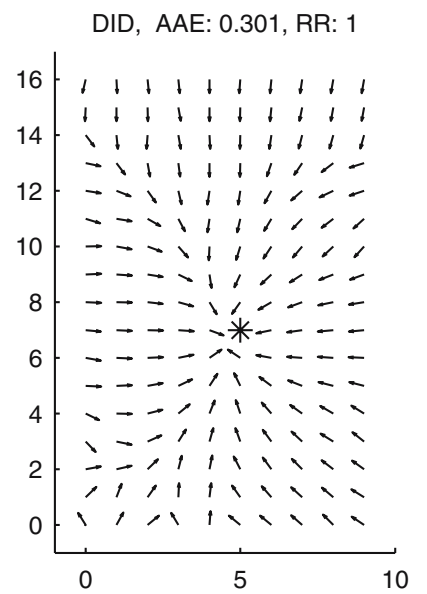

DID, AAE: 1.14, RR: 0.195

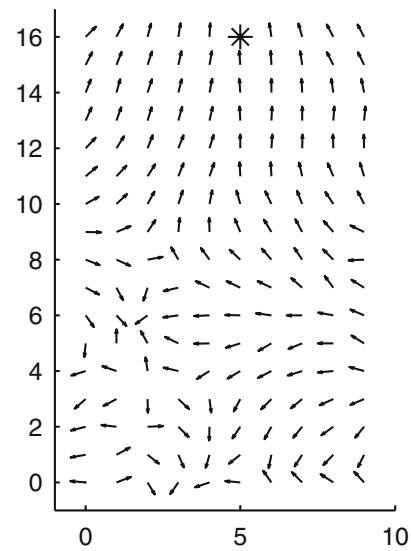

MFDID, AAE: 0.178, RR: 1

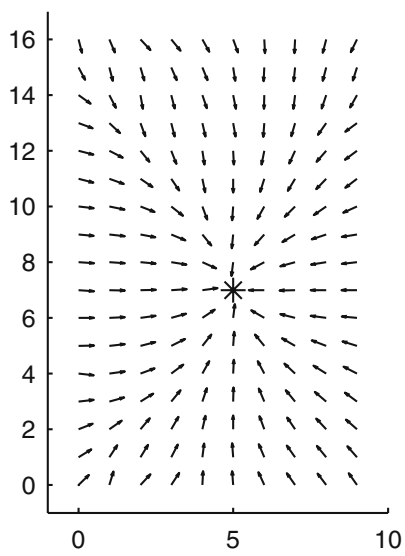

MFDID, AAE: 1.73 , RR: 0.231

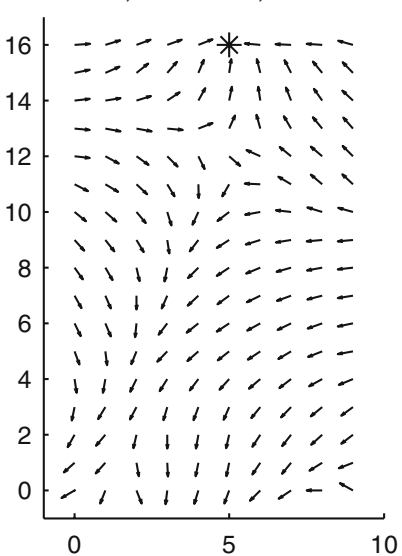

FirstOrder, AAE: 0.236, RR: 1

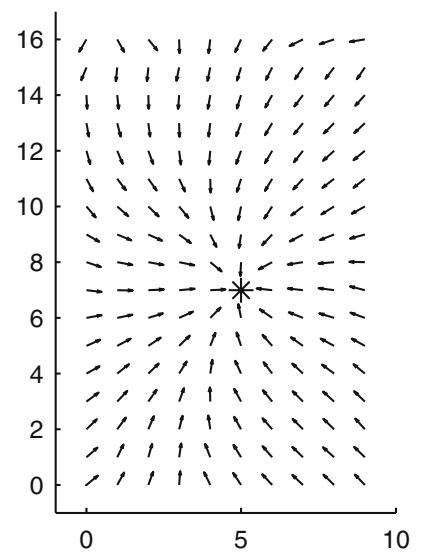

FirstOrder, AAE: 0.26, RR: 1

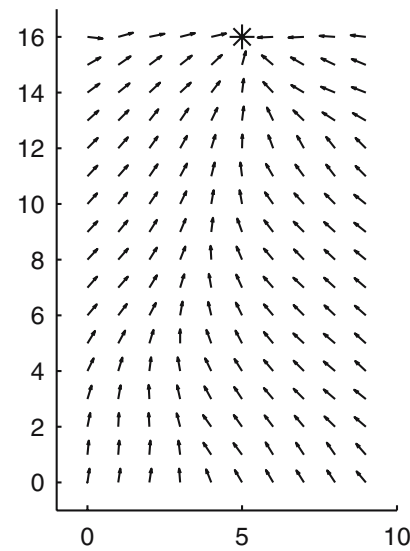

Fig. 6 Home vector fields for goal positions $(5,7)$ (top row) and $(5,16)$ (bottom row) in lab environment

structure. The absence of a sharply defined attractor point at the goal, as well as the presence of local minima, implies that homing to position $(5,16)$ will not be successful for distant starting positions. In the results presented above we found poor performance for both DID and MFDID along the line $y=16$.

The structure of the distance function in this region of the capture grid is likely due to the appearance of the table lying along the top wall of the lab. ${ }^{2}$ At $y=16$, the robot's camera was practically underneath this table; therefore the top wall was occluded by the darker underside of the table. However, for $y<16$ the white wall above the table is more prevalent. Compare the top two images in the left-hand column of Fig. 10 to the bottom two to see this effect. The impact on the shape of the image distance function is such that DID and MFDID are relatively ineffective for goals in this region.

One result that may be surprising is the fact that MFDID consistently outperformed DID. One might suppose that DID would be the better performer given

\footnotetext{
2 We refer to this as the "top wall" as it corresponds to the top of the plots in Figs. 6 and 7.
}

that it actually samples the image distance function, while MFDID only approximates this function. Further, MFDID relies upon an equal-distance assumption that will clearly be unsatisfied in many environments - particularly in the hall environment where MFDID actually performs quite well. We believe that the superior performance of MFDID lies in yet another factor. To estimate the gradient of the image distance function, DID relies on images taken from two adjacent positions in the capture grid. Both of these images are taken from positions $30 \mathrm{~cm}$ from the current position. MFDID, on the other hand, estimates the gradient using two synthetic images taken from infinitesimally close positions. Thus, the gradient determined by MFDID is more sensitive to the fine local structure of the approximated distance function. To ascertain the impact of this factor, we tested DID with increasing step sizes (multiples of $30 \mathrm{~cm}$ ). The results shown in Fig. 11 indicate that the optimal step size for DID is less than 1 . Such a step size is not achievable with this database. It may be the case that for online control of a mobile robot, DID could match the performance of MFDID by decreasing its step size. 

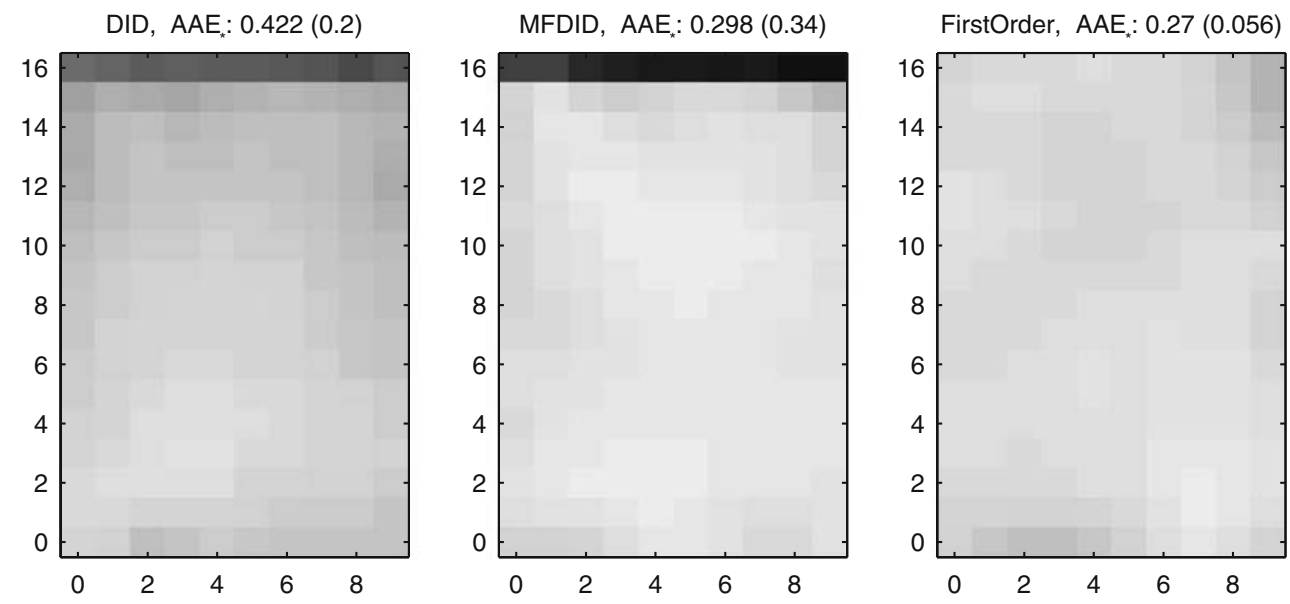

DID, RR: $0.932(0.21)$
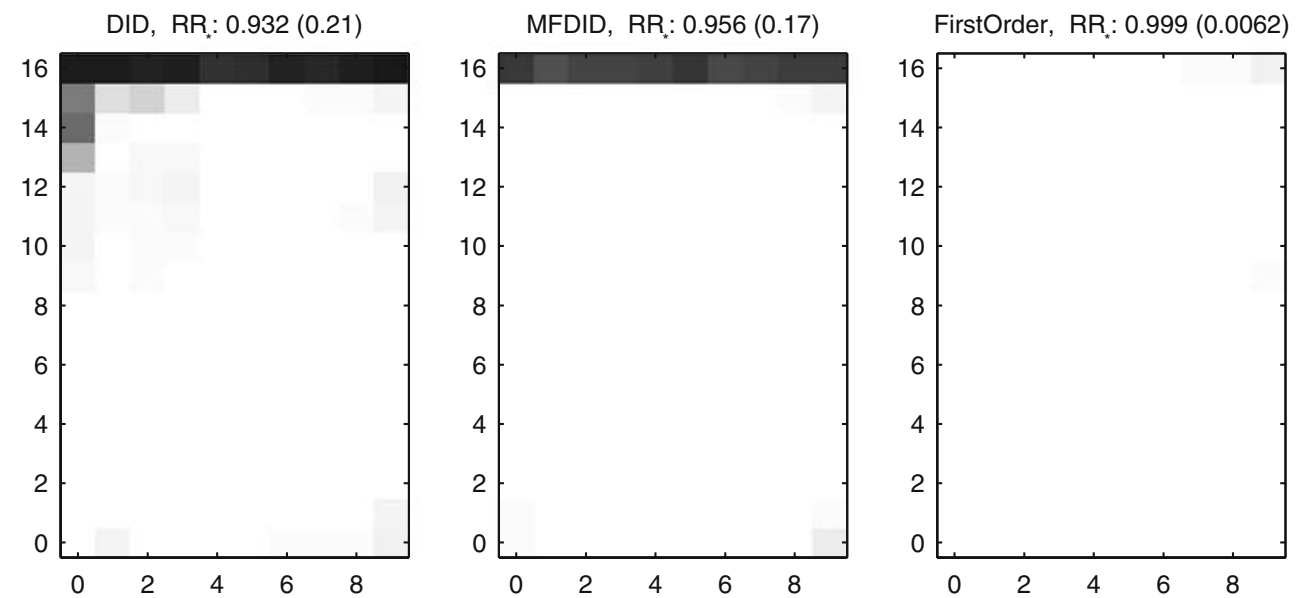

Fig. 7 AAE and RR for all goal positions in lab environment. White represents an AAE value of 0 , or an RR value of 1 . Black represents an AAE value of 1.8333 (maximum), or an RR value

A possible reason to explain the difference in performance between MFDID and the differential flow method is the underestimation of vertical flow vector components close to the horizon (Sect. 3). Generally, we found that the differential methods performed well in the lab environment whereas MFDID performed well in the hall environment. Images from the hall environment, as shown in Fig. 10, are dominated by vertical structures. These vertical structures will tend to exhibit mostly horizontal flow vectors. Thus, in the hall environment, MFDID's lack of emphasis on vertical flow does not hinder performance relative to FirstOrder. Images from the lab environment, on the other hand, include a great number of horizontal structures, which will tend to exhibit mostly vertical flow vectors.

\subsection{Descent methods}

In the following, we discuss the MFDID method and introduce two versions that require only a single flow template rather than two. of 0 . The numbers above each plot show the mean and standard deviation (in parentheses)

Homing with two flow templates The MFDID method suggested in this paper derives from a version of the original DID method where the gradient is estimated by sampling the distance measure in two perpendicular directions. We assumed that these test steps originated from the same current location; in a practical application, the robot could sample two points at the beginning and end of a forward movement, and the third point after $\mathrm{a} \pm 90^{\circ}$ turn and a subsequent forward movement, preferably of equal length. The gradient would be computed at the second point of this sequence, but applied at the third. Now the core idea of the MFDID is to replace the two test steps with predictions of how the image would change under two small movements.

Each prediction is based on a "matched filter" or template flow field for a translation in the corresponding direction. A matched filter is a concept in neuroethology and describes the spatial layout of some population of receptors that is matched to a certain aspect of the task (Wehner 1987). For example, preferred directions for arrays of elementary motion detectors seem to corre- 

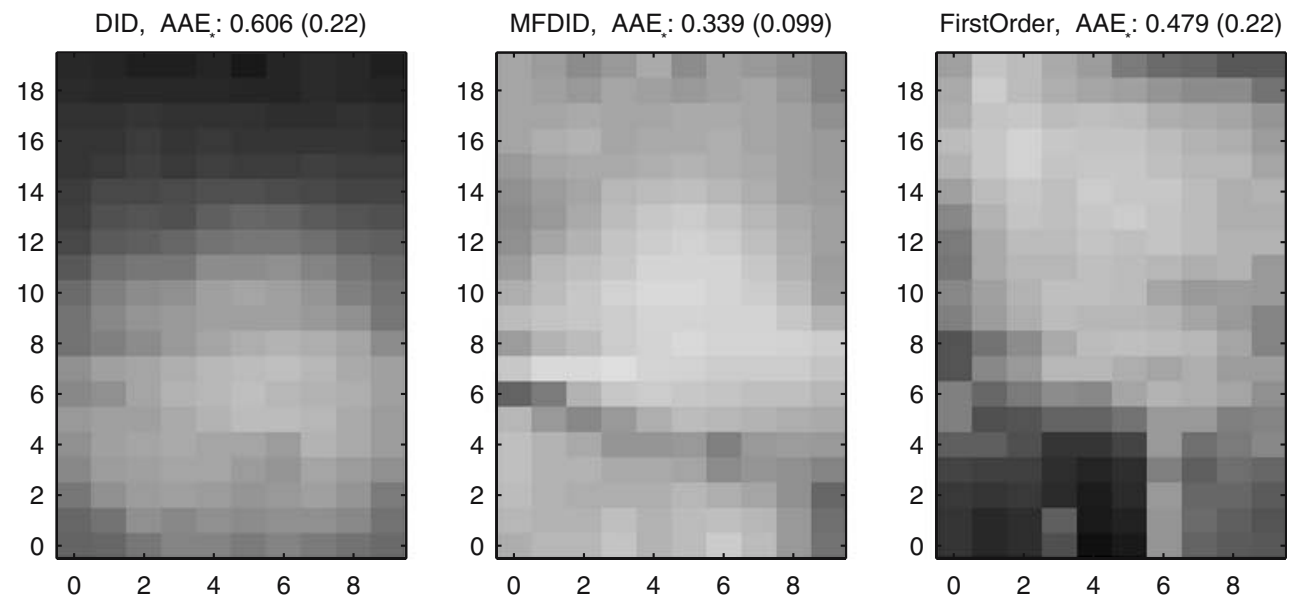

DID, RR: $0.751(0.21)$
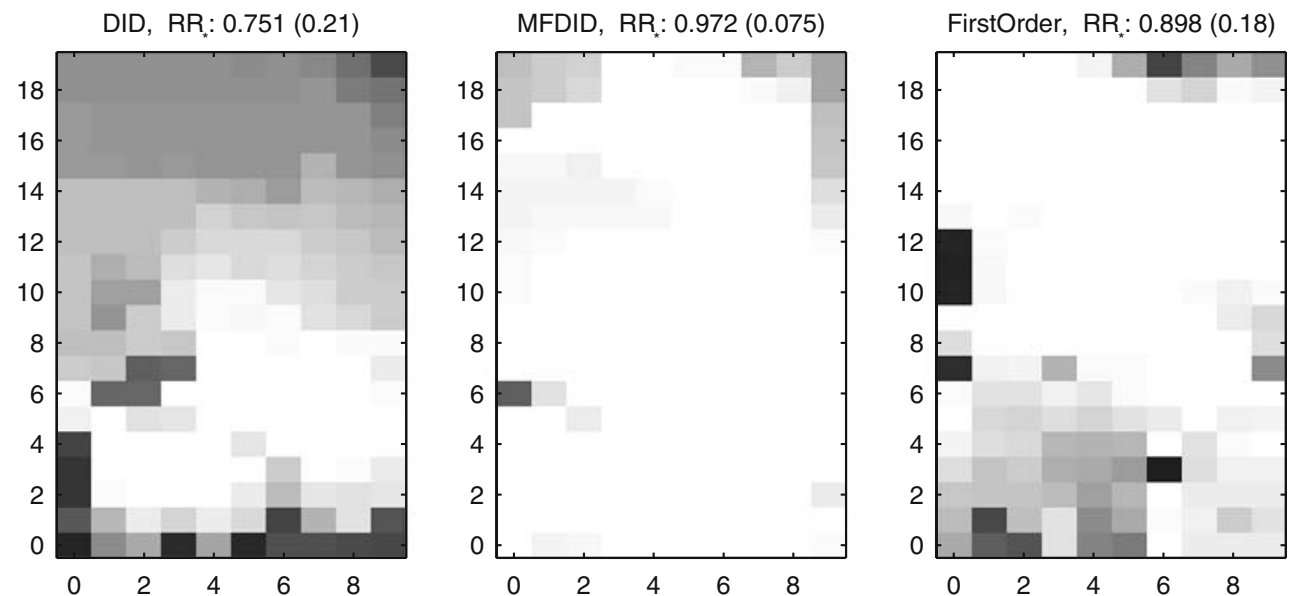

Fig. 8 AAE and RR for all goal positions in hall environment. White represents an AAE value of 0, or an RR value of 1. Black represents an AAE value of 1.1343 (maximum), or an RR value of 0 . Notation as for Fig. 7

spond to flow fields induced by particular movements of flies, e.g., by roll movements (Egelhaaf et al. 2002; Krapp 2000; Franz and Krapp 2000; Franz et al. 2004).

Our matched filters have the typical structure of flow fields for pure translations in the horizontal plane: a focus of expansion, a focus of contraction, and nearly horizontal flow between them (Fig. 4). If no depth information is available, though, only the directions of the flow vectors in the two matched filters are known, but not their length. Our prediction therefore rests on an "equal-distance assumption" as the warping method (Franz et al. 1998b). The performance of the MFDID method shows that, as in the warping method, even severe violations of this assumption have only mild effects on the home vector direction.

In the MFDID method in Eq. (12), the intensity gradient of the current image is projected onto the two matched filters (the rows of $\mathbf{B}^{\mathrm{T}} \boldsymbol{\Gamma}$ ) and multiplied with the difference between the intensity of the pixel in the snapshot and in the current view. This is essentially a comparison between the intensity change in the direc- tion of the flow vector and the change that would be required to become more similar to the snapshot. If the signs of the two factors coincide, a movement in the direction corresponding to the flow template would make this pixel in the current view more similar to the same pixel in the snapshot. In this case, the local home vector component would be positive (pointing in the template's corresponding movement direction).

Homing with a single frontal flow template We mentioned above that a matched filter is supposed to correspond to the flow experienced under some typical movement of the animal. In what follows we assume that the matched filters are bound to the agent's coordinate system, with a "frontal" filter where the focus of expansion coincides with the usual movement direction and a "sideways" filter where the foci of expansion and contraction are at $\pm 90^{\circ}$ from the movement direction. While the frontal filter will relate to flow experienced under forward translations, the sideways filter is less plausible, at least for walking animals like ants. Moreover, the frontal filter may simultaneously serve 
Fig. 9 Performance of all methods for varying cutoff frequencies (lab environment)
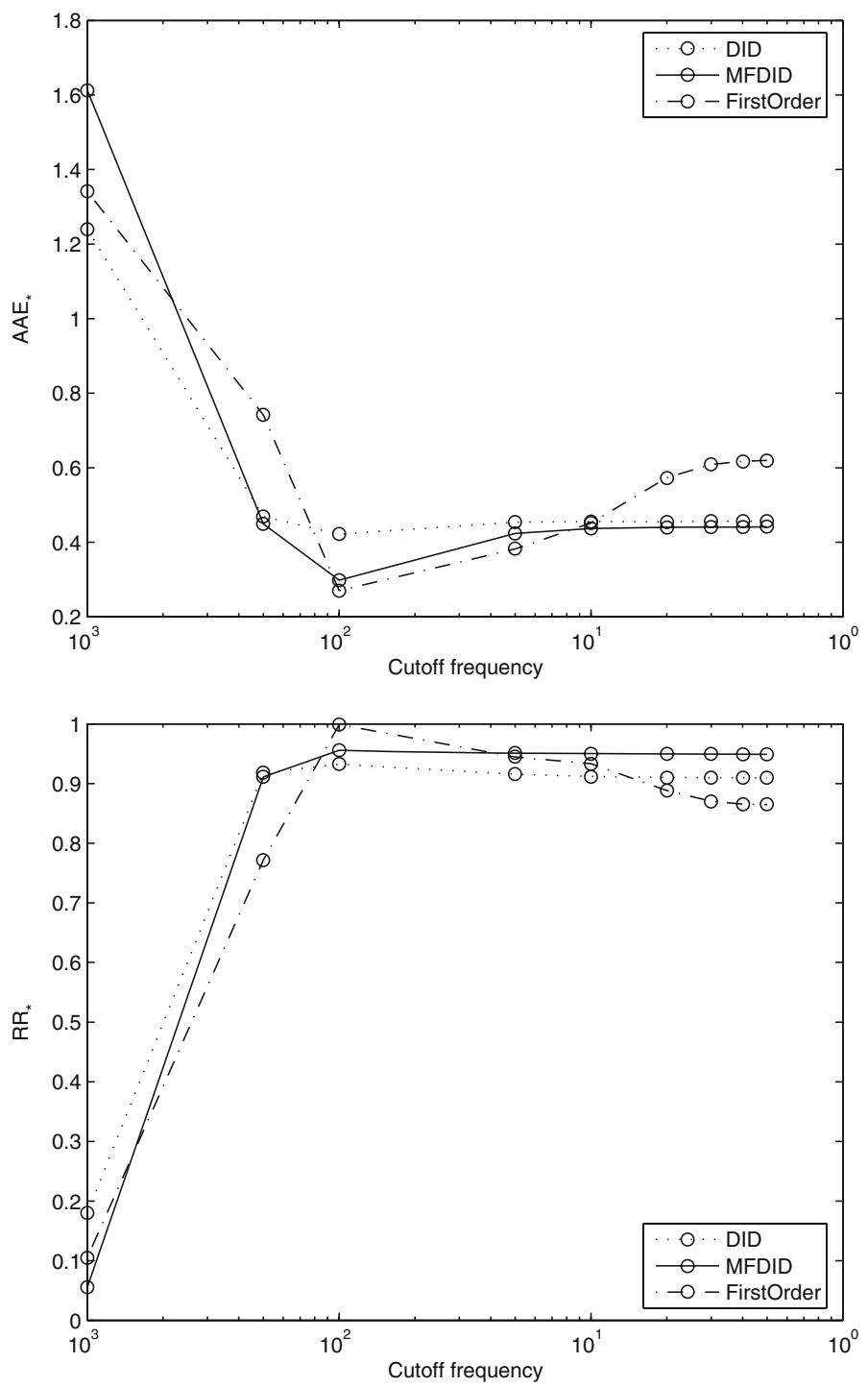

$H(\alpha, \mathbf{x})=\left(\begin{array}{c}\cos \alpha \\ \sin \alpha\end{array}\right)^{\mathrm{T}} \cdot \mathbf{h}(\mathbf{x})$.

some other purpose like path integration; at least for walking insects, it is more difficult to come up with an alternative purpose for the sideways filter.

In what follows we therefore briefly discuss a version of MFDID where a single matched filter in forward direction is sufficient. We start from the fact that the gradient is the direction with the largest directional derivative and from the assumption that the directional derivative will vary smoothly with varying gaze direction. Probing the directional derivative could be accomplished by small head rotations or small changes in the orientation of the body. For each gaze direction, the directional derivative can be determined by applying just the frontal flow field in the same way as described above for the MFDID method. Turning toward larger values will then gradually align the animal with the home direction.

For our case of gradient descent, we can define the directional derivative as
Thus, the directional derivative is the projection of the home vector onto a vector pointing in direction $\alpha$. We now give up the assumption that the agent's visual coordinate system is aligned with the world coordinates irrespective of its body orientation, but instead assume that the current view is bound to the body of the agent. (For all methods discussed so far, the snapshot would have to be mentally rotated to an orientation matching that of the current view.) Then we can fix the template flow field in the movement direction of the agent's coordinate system $\alpha=0$. We also insert $\mathbf{h}(\mathbf{x})$ from Eq. (10) and obtain

$$
\begin{aligned}
H(0, \mathbf{x})= & \sum_{i, j} \frac{1}{D} \mathbf{e}_{1}^{\mathrm{T}} \mathbf{B}\left(\beta_{i}\right)^{\mathrm{T}} \boldsymbol{\Gamma}\left(\gamma_{j}\right) \cdot \nabla_{\varphi} C\left(\boldsymbol{\varphi}_{i j}, \mathbf{x}\right) \\
& \times\left[C\left(\boldsymbol{\varphi}_{i j}, \mathbf{x}\right)-S\left(\boldsymbol{\varphi}_{i j}\right)\right] .
\end{aligned}
$$




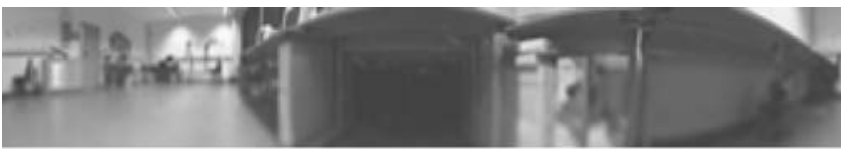

$(0,16)$

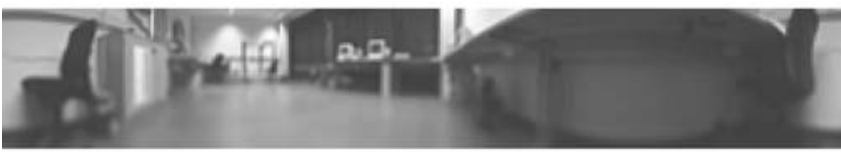

$(9,16)$

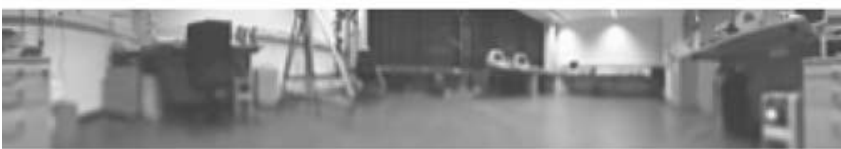

$(9,0)$

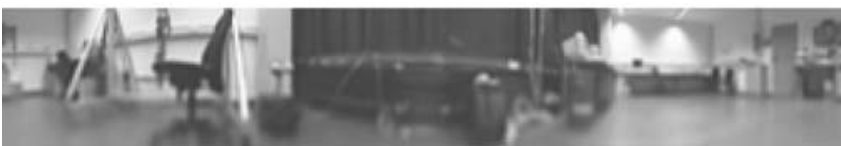

$(0,0)$

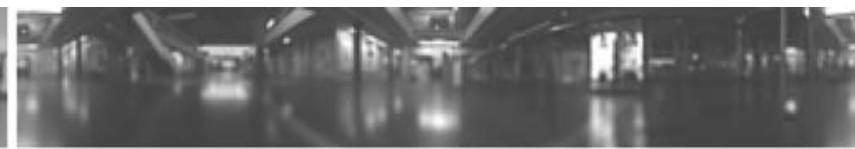

$(0,19)$

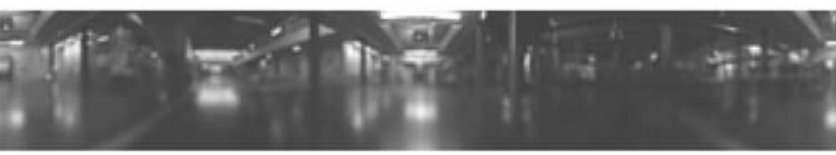

$(9,19)$

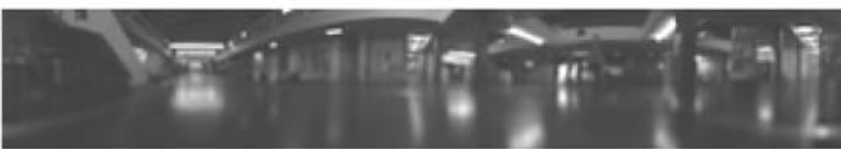

$(9,0)$

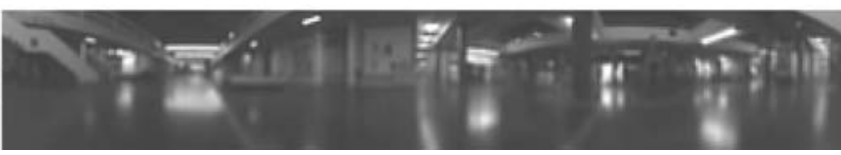

$(0,0)$

Fig. 10 Images from four corners of lab environment (left column) and hall environment (right column). These images were obtained by low-pass filtering (cutoff 0.1, order 3) and unfolding as described in Sect. 4.1

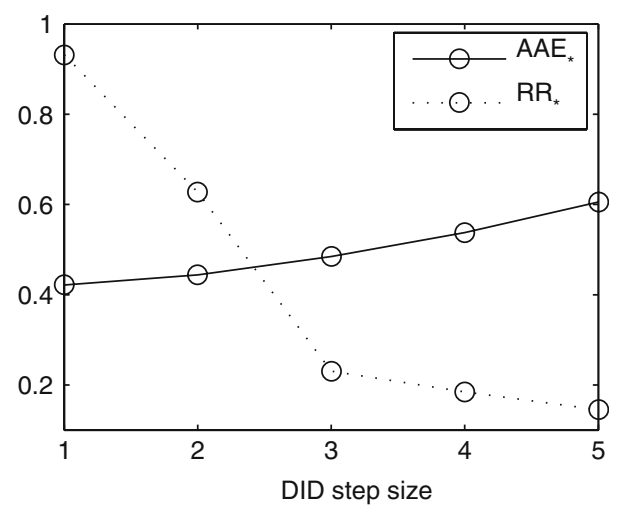

Fig. 11 Performance of DID (lab environment) for varying step size $s$; see Eq. (17)

By comparing this equation to the home vector in Eq. (10), we see that $H(0, \mathbf{x})$ is just a projection onto the frontal flow filter (focus of expansion in the direction of movement): The intensity gradient is projected onto the first row of $\mathbf{B}^{\mathrm{T}} \boldsymbol{\Gamma}$. By looking into different directions, e.g., just by moving the head slightly from side to side, and predicting the image change through the frontal flow filter, the agent can detect a turn direction in which the directional derivative increases. Figure 12 (center) shows the length of the directional derivative computed in this way for three current locations in different directions.

Of course, a similar strategy could also be applied without prediction by actually measuring the change of the SSE under small translations. Rather than sampling the SSE at three points in a right triangle, the agent would move on a slightly winding trajectory. After having executed a small translatory movement, the agent determines an approximation of the directional derivative from SSE values sampled at the beginning and the end of this step, changes the heading angle by a small amount, executes another translatory movement, again approximates the directional derivative from two SSE values, and then decides what direction to turn in so that the directional derivative will decrease. This method may be problematic, though, since the two translatory steps do not originate from the same point.

Homing with a single sideways flow template In the method described in the previous paragraph, the agent will turn toward an increasing directional derivative. This opens an alternative approach to MFDID that has the advantage of not requiring any sampling movements (not even changes in gaze direction) but that is less plausible with respect to the matched-filter concept since at least walking animals will rarely experience this flow field. In this approach the agent will not determine a home vector directly but will determine an amount by which to turn at each step. The agent can turn according to the derivative of the directional derivative $H(\alpha, \mathbf{x})$

$\frac{\mathrm{d} \alpha}{\mathrm{d} t}=\varepsilon \frac{\partial H(\alpha, \mathbf{x})}{\partial \alpha}$,

where the term on the r.h.s. can simply be determined from Eq. (20): 


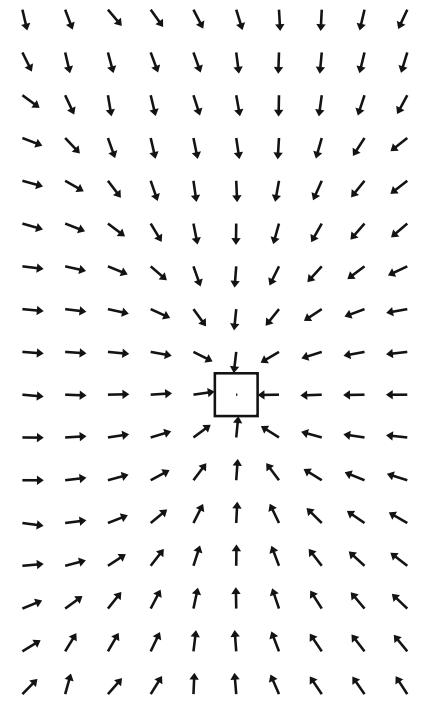

Fig. 12 MFDID method applied to lab database. Left Home vector field for goal location in center of black square, obtained by applying two flow templates. Center Directional derivative for different gaze directions computed at three different locations, determined from a single frontal flow template. The directional derivative is represented by the length of the gray vectors pointing in the gaze direction. The black vector is the home vector. Right

$\frac{\partial H(\alpha, \mathbf{x})}{\partial \alpha}=\left(\begin{array}{c}-\sin \alpha \\ \cos \alpha\end{array}\right)^{\mathrm{T}} \cdot \mathbf{h}(\mathbf{x})$.

Again, we change from fixed coordinates to agent-bound coordinates $(\alpha=0)$ and insert the home vector from Eq. (10), which yields

$$
\begin{aligned}
\frac{\partial H(0, \mathbf{x})}{\partial \alpha}= & \sum_{i, j} \frac{1}{D} \mathbf{e}_{2}^{\mathrm{T}} \mathbf{B}\left(\beta_{i}\right)^{\mathrm{T}} \boldsymbol{\Gamma}\left(\gamma_{j}\right) \cdot \nabla_{\varphi} C\left(\boldsymbol{\varphi}_{i j}, \mathbf{x}\right) \\
& \times\left[C\left(\boldsymbol{\varphi}_{i j}, \mathbf{x}\right)-S\left(\boldsymbol{\varphi}_{i j}\right)\right] .
\end{aligned}
$$

A comparison with the home vector in Eq. (10) reveals that this equation projects the intensity gradient onto the sideways flow filter (second row of $\mathbf{B}^{\mathrm{T}} \boldsymbol{\Gamma}$ ). In this filter, the foci of expansion and contraction lie at $\pm 90^{\circ}$ from the forward direction. The behavior of this method is shown in Fig. 12 (right): Starting from three different points in two different orientations, the agent is turning toward the snapshot location and approaching this location while moving with constant speed. For the sake of demonstration, we chose a small value for $\varepsilon$; this leads to slow turning and exaggerates the differences between the two trajectories originating at the same point.

Since it requires only a single matched filter and has no motor components (no head movements, no translational test steps), this method is clearly attractive for applications where the robot's task is to approach a target location. The method has its limitations when the local homing method is integrated into topologi-
Trajectories produced by determining the turn direction from a single sideways flow template. From each of the three starting points (black dots), the simulated agent starts with two different initial orientations (looking to the left side of the grid or to the right). The closest view in the database grid to the agent's position is used for the computation. The agent moves in steps of 0.3 grid units

cal navigation in a view graph (Franz et al. 1998a) since it does not provide a home vector. For some purposes, for example to select a new direction of exploration at a graph node, it is more efficient if the home vector toward neighboring graph nodes can be determined directly. The method with the single sideways filter would have to physically rotate the robot (or the flow template) in the home direction. Here the MFDID method using two matched filters is more efficient.

\subsection{Other matched-filter methods}

In the main part of this paper we derived and tested the MFDID method where the matched-filter concept is applied to simplify the DID suggested by Zeil et al. (2003). We focused on the DID method because of its simplicity and low computational complexity. However, the matched-filter approach is a more general concept and can be applied to derive closed-form solutions for all methods that are formulated as a gradient descent in a distance measure. With the three methods presented in what follows we intend to illustrate the broader applicability of the matched-filter concept. The first two methods adhere to the concept of DID but use a different distance measure and a different template flow field, respectively, whereas in the third example we derive the matched-filter solution for the Fourier-amplitude method (Menegatti et al. 2004). 
MFDID with different distance measure The DID method is based on the RMS error in Eq. (1) or the SSE in Eq. (2) between images, but a number of alternative distance measures could be used as well; Giachetti (2000) gives an overview in the context of block matching. One might conjecture, for example, that the covariance measure

$$
\begin{aligned}
p(\mathbf{x}) & =\operatorname{COV}(\mathbf{x}) \\
& =\frac{1}{N} \sum_{i, j}\left[C\left(\boldsymbol{\varphi}_{i j}, \mathbf{x}\right)-\bar{C}(\mathbf{x})\right] \cdot\left[S\left(\boldsymbol{\varphi}_{i j}\right)-\bar{S}\right]
\end{aligned}
$$

(where $N$ is the total number of pixels and the overlined quantities are average image intensities) is more robust against global variations of brightness than RMS and SSE. It is straightforward to derive the home vector for this method by approximating the spatial gradient with the intensity gradient according to Eq. (9):

$$
\begin{aligned}
\mathbf{h}(\mathbf{x})= & -\frac{1}{N} \sum_{i, j} \frac{1}{D} \mathbf{B}\left(\beta_{i}\right)^{\mathrm{T}} \boldsymbol{\Gamma}\left(\gamma_{j}\right) \\
& \times \nabla_{\varphi} C\left(\boldsymbol{\varphi}_{i j}, \mathbf{x}\right) \cdot\left[S\left(\boldsymbol{\varphi}_{i j}\right)-\bar{S}\right] .
\end{aligned}
$$

(Note that in this case the home vector is obtained from a gradient ascent in the potential.) While MFDID with the SSE as distance measure produces a home vector that may appear to be just a version of the first-order method with a different weighting of the flow vectors [compare Eq. (18) with Eq. (19)], other distance measures result in solutions such as Eq. (21) that are more remotely related to the first-order flow method.

MFDID with rotational flow template Both the original MFDID method given by Eq. (10) and the method derived from the covariance measure given by Eq. (21) use two purely translational flow templates to approximate the gradient. These methods are based on the observation by Zeil et al. (2003) that the image distance varies smoothly over the spatial distance to the goal. The same authors report that the image distance also changes smoothly when images from two nearby locations are rotated against each other. The distance measure exhibits a pronounced minimum at the angle where the two images are approximately aligned as if they had been captured in the same camera orientation. The rotation angle can be found either by rotating the images through all angles and searching for the minimal SSE or by a gradient descent in the rotation angle $\alpha$ with respect to the SSE. We can apply the matched-filter approach to the gradient descent by formulating the distance

$p(\alpha)=\operatorname{SSE}(\alpha)=\frac{1}{2} \sum_{i, j}\left[C\left(\varphi_{i j}, \alpha\right)-S\left(\varphi_{i j}\right)\right]^{2}$.
Here we omitted the position $\mathbf{x}$ of the current view and introduced the rotation angle $\alpha$ as parameter. In this case, our template flow field corresponds to a pure rotation around the vertical axis, i.e., we insert $\dot{\mathbf{x}}=\mathbf{0}$ and $\omega=\omega(0,0,1)^{\mathrm{T}}$ into the flow Eq. (5). After some manipulations, we obtain the descent equation over the rotation angle $\alpha$ :

$\dot{\alpha}=-\sum_{i, j} \frac{\mathrm{d} C\left(\varphi_{i j}, \alpha\right)}{d \beta_{i}} \cdot\left[C\left(\varphi_{i j}, \alpha\right)-S\left(\varphi_{i j}\right)\right]$.

If the agent rotates according to $\dot{\alpha}$, the image distance between the current view and the snapshot is reduced. Note that the test steps for rotation come practically for free (the image can be rotated without movements), so this method does not share with MFDID the advantage of saving test steps; we are mainly presenting this for the sake of demonstrating the general applicability of the matched-filter method.

Fourier-amplitude method In the Fourier-amplitude method introduced by Menegatti et al. (2004), each image is characterized by the first $K$ amplitudes of the Fourier spectra of each row of the panoramic image. Since Fourier amplitudes are invariant against shifts, this method does not require a compass to align the two images before computing the distance. We denote the discrete Fourier transform as

$F_{k}(I)=\frac{1}{\sqrt{n}} \sum_{i=0}^{n-1} I_{i} \exp \left(-\imath k i \frac{2 \pi}{n}\right)$,

where $n$ is the number of pixels per image row, $I_{i}$ is pixel $i$ of the image row $I$, and $l$ is the imaginary unit (see, e.g., Jähne 2002). As the distance measure we use the SSE applied to the Fourier amplitudes of corresponding rows $j$ in the two images, a simple formulation would be

$p(\mathbf{x})=\frac{1}{2} \sum_{j} \sum_{k=-K}^{K}\left(\left|F_{k}\left(C_{j}(\mathbf{x})\right)\right|-\left|F_{k}\left(S_{j}\right)\right|\right)^{2}$.

Here, $C_{j}(\mathbf{x})$ and $S_{j}$ denote row $j$ of the panoramic images. We introduce the two components of the spatial gradient that is approximated by the intensity gradient according to Eq. (9):

$$
\left(\begin{array}{l}
\Phi_{i j}(\mathbf{x}) \\
\Psi_{i j}(\mathbf{x})
\end{array}\right)=\frac{\delta}{D} \mathbf{B}\left(\beta_{i}\right)^{\mathrm{T}} \boldsymbol{\Gamma}\left(\gamma_{j}\right) \nabla_{i j} C_{i j}(\mathbf{x}),
$$

with $\delta$ from Eq. (11). The home vector equation is derived in the usual way. We use the abbreviations $\Re_{k}(I)=\operatorname{Re}\left\{F_{k}(I)\right\}$ and $\Im_{k}(I)=\operatorname{Im}\left\{F_{k}(I)\right\}$ and omit the argument $\mathbf{x}$ : 


$$
\begin{aligned}
\mathbf{h}= & -\sum_{j} \sum_{k=-K}^{K}\left|F_{k}\left(C_{j}\right)\right|^{-1} \\
& \times\left(\begin{array}{l}
\Re_{k}\left(\Phi_{j}\right) \Re_{k}\left(C_{j}\right)+\Im_{k}\left(\Phi_{j}\right) \Im_{k}\left(C_{j}\right) \\
\Re_{k}\left(\Psi_{j}\right) \Re_{k}\left(C_{j}\right)+\Im_{k}\left(\Psi_{j}\right) \Im_{k}\left(C_{j}\right)
\end{array}\right) \\
& \times\left(\left|F_{k}\left(C_{j}\right)\right|-\left|F_{k}\left(S_{j}\right)\right|\right) .
\end{aligned}
$$

The method requires a discrete Fourier transform of each row $j$ in images $C, S, \Phi$, and $\Psi$.

\subsection{MFDID versus warping}

Both our approach and the warping method introduced by Franz et al. (1998b) use matched filters for local visual homing. In what follows, we highlight the differences between the two methods. Formulated in the context of matched filters, warping applies a large number of different flow templates to one of the images (e.g., the current view). The templates correspond to movements with three parameters describing two rotations and a translation. The rotation parameters cover the full range of $360^{\circ}$ each and the translation parameter essentially varies between zero and the average distance to the landmarks (with a typical angular resolution of $10^{\circ}$ and 20 steps for the distance, 25,920 templates are required). The distorted images obtained by passing the current view through the templates are compared to the snapshot by some distance measure (e.g., SSE). The home vector can then be expressed by the parameters that were used to produce the best-matching distorted current view. It is an advantage of the warping method that no compass is necessary since the robot rotation is covered by the two rotational parameters. Warping can in principle be applied to 2D images but is computationally feasible only for $1 \mathrm{D}$ images (for our image size of $300 \times 50$ and the parameter resolution given above, a single home vector computation would require ca. $4 \cdot 10^{9}$ operations). To summarize, warping is a search procedure where one image is explicitly distorted according to a large number of templates and compared to the other image by some distance measure. The templates are describing all possible and thus also large movements from the current position to the goal position.

In contrast, the MFDID method derived in this paper is using just two flow templates for translational movements. The derivation is based on the assumption that these movements are infinitesimally small. The image distortion is performed implicitly: Rather than computing the change of the image and the corresponding change in the distance measure, both steps are fused in a single equation that directly provides the home vector. MFDID performs no search and its complexity therefore scales linearly with the number of pixels in the image; this also makes the application to 2D images practically feasible. MFDID requires a compass to align the two images to the same coordinate system which is a clear disadvantage compared to warping.

Both methods are based on the assumption that all features have approximately the same distance from the camera. For the warping method it could be proved mathematically that the error due to this assumption decreases when the agent approaches the goal (Franz et al. 1998b); whether this property also holds for MFDID is still an open question. In the practical application, violations of the equal distance assumption appear to have only mild effects on the overall performance of either warping and MFDID.

\section{Conclusions}

MFDID replaces exploratory movements for the estimation of the gradient by two image predictions. The predictions are based on matched flow filters for translatory movements in the plane. The method is closely related to differential optical flow methods and exhibits comparable performance; the major difference lies in the influence of vertical flow components close to the horizon. In our image database experiments, MFDID performed better than the original DID method with perpendicular test steps, despite the underlying equaldistance assumption, although this may be due to the coarse resolution of the database grid. We show that it is also possible to navigate either with a single frontal flow template and exploratory changes in gaze direction or with a single sideways flow template without any exploratory movements. The matched-filter approach can generally be applied to gradient DID measures and thus also opens a new perspective on parameter models of local visual homing.

Acknowledgments R. Möller is grateful to Axel Könies for his help in shortening the derivation of the MFDID method. Applying the matched-filter concept to the parameter method by Menegatti et al. (2004) was a suggestion by Sebastian Fischer.

\section{References}

Barron JL, Fleet DJ, Beauchemin SS (1994) Performance of optical flow techniques. Int J Comput Vis 12:43-77

Batschelet E (1981) Circular statistics in biology. Academic, San Diego

Beauchemin SS, Barron JL (1995) The computation of optical flow. ACM Comput Surv 27:433-467

Cartwright BA, Collett TS (1983) Landmark learning in bees. J Comp Physiol A 151:521-543 
Egelhaaf M et al (2002) Neural encoding of behaviorally relevant visual-motion information in the fly. Trends Neurosci 25:96-102

Franz MO, Chahl JS, Krapp HG (2004) Insect-inspired estimation of egomotion. Neural Comput 16:2245-2260

Franz MO, Krapp HG (2000) Wide-field, motion-sensitive neurons and matched filters for optic flow fields. Biol Cybern 83:185-197

Franz MO, Mallot HA (2000) Biomimetic robot navigation. Robot Auton Syst, special issue: Biomimetic Robots 30:133-153

Franz MO, Schölkopf B, Mallot HA, Bülthoff HH (1998a) Learning view graphs for robot navigation. Auton Robots 5:111-125

Franz MO, Schölkopf B, Mallot HA, Bülthoff HH (1998b) Where did I take that snapshot? Scene-based homing by image matching. Biol Cybern 79:191-202

Giachetti A (2000) Matching techniques to compute image motion. Image Vis Comput 18:247-260

Jähne B (2002) Digital image processing, 5th edn. Springer, Berlin Heidelberg New York

Koenderink JJ, van Doorn AJ (1987) Facts on optic flow. Biol Cybern 56:247-254

Krapp HG (2000) Neuronal matched filters for optic flow processing in flying insects. Int Rev Neurobiol 44:93-120

Lambrinos D, Möller R, Labhart T, Pfeifer R, Wehner R (2000) A mobile robot employing insect strategies for navigation. Robot Auton Syst, special issue: Biomimetic Robots 30: 39-64
Menegatti E, Maeda T, Ishiguro H (2004) Image-based memory for robot navigation using properties of omnidirectional images. Robot Auton Syst 47:251-267

Möller R (2000) Insect visual homing strategies in a robot with analog processing. Biol Cybern 83:231-243

Möller R (2001) Do insects use templates or parameters for landmark navigation? J Theor Biol 210:33-45

Möller R (2002) Visual homing without image matching. In: Ayers J, Davis JL, Rudolph A (eds) Neurotechnology for biomimetic Robots. MIT Press, Cambridge, pp 517-532

Stürzl W, Mallot HA (2002) Vision-based homing with a panoramic stereo sensor. In: Biologically motivated computer vision. Lecture Notes in Computer Science 2525. Springer, Berlin Heidelberg New York, pp 620-628

Stürzl W, Mallot HA (2006) Efficient visual homing based on Fourier transformed panoramic images. Robot Auton Syst 54:300-313

Vardy A, Möller R (2005) Biologically plausible visual homing methods based on optical flow techniques. Connect Sci 17:47-89

Wehner R (1987) 'Matched filters' - neural models of the external world. J Comp Physiol A 161:511-531

Wehner R, Räber F (1979) Visual spatial memory in desert ants, cataglyphis bicolor (Hymenoptera: Formicidae). Experientia 35:1569-1571

Zeil J, Hoffmann MI, Chahl JS (2003) Catchment areas of panoramic images in outdoor scenes. J Opt Soc Am A 20:450-469 